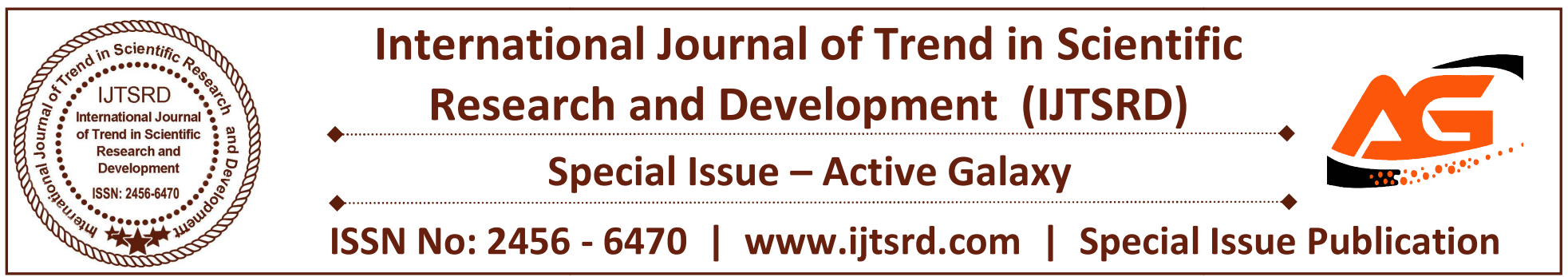

\title{
A Novel Colour Image Embedded QR Code Generation Technique for Chip-Less RFID Labelling
}

\author{
Dr S Hemalatha ${ }^{1}$, R latha ${ }^{2}$, S Mythili ${ }^{3}$ \\ ${ }^{1}$ Professor, ${ }^{2}$ Assistant Professor, ${ }^{3}$ Student \\ Veltech Hightech Dr Rangarajan Dr Sakunthala Engineering College, \\ Avadi, Chennai, India
}

\begin{abstract}
In recent years, chip less RFID mark located based on genetic algorithm (GA) optimization system is used. The genetic algorithm is fit and facility to arrangement a band of frequency domain chippers card like QR display. The resultant card supports an extend $30 \mathrm{~mm} \times 30 \mathrm{~mm}$ and reside of lattice limited metallic pieces arranged randomly over a $60 \times 60$ bit design frame a collection of advanced structured. The frequency signature of the GA-based mark is optimized to consistent with an FSK-based ciphering process and a magnitude of 8 segments is acquired. The construct of the resultant mark is proven. Optimum mark model made in silver-ink, marked down flexible bases, serigraphy, which is a largeconsistent performance procedure. The possibility of this optimization system for the pattern of chip less RFID label is affirmed.
\end{abstract}

Keywords: Genetic algorithm (GA), chipless RFID, quick response code (QR).

\section{Introduction}

A QR code is a 2 dimensional structure. It was initially created in 1994 by Denso Wave. A method to route vehicles they collected, and to scan elements at speedy. The data is designed to cipher information in horizontal and vertical way, thus dimension up to certain periods enormous information than a customary bar code. QR codes have directly advanced as used for ID verification in medical, recording and track, car manufacturer, and marketing activities. The original form was $21 \times 21$ elements and retained just 4 quality value of information. They are forty distinct form of QR Codes primarily peculiar in terms of number of units. The large QR Code (Version 40) has a capacity of $177 \times 177$ data section can store up to 23,648 info. Error improvement in QR Codes is supported on Reed-Solomon Coder. The main objective is to embed QR codes to color images. The notion of $\mathrm{QR}$ images, a determined technique to embed QR codes into color images with less hazard of noticing mistake. This technique can be practically utilized for color image. It has corrected resolution and data. These are beginning with safety, car spare parts manufacturing, maintenance, telecommunication and many industries, and mobile phones. QR code read data from any angle.

The rest of the chapters are organized as foolows: Chapter II deals with the related works on QR Coding, chaoter III presented about QR Code basics and GA, Chapter IV proposed a new architecure, Chapter V explained with the embedding QR code in to colour image and finally CHpter VI Concludes the work.

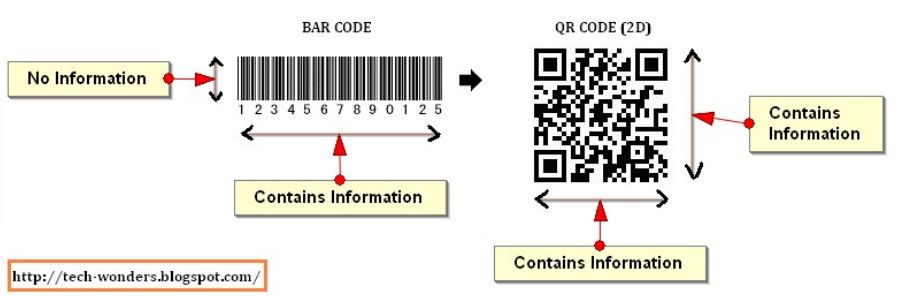

Fig 1: storing information 


\section{RELATED WORKS}

An RFID integrated QR code antenna can provide extended security features by employing two technologies (QR code + RFID) together. These features can be used in a combined or independent manner. The tag antenna part can be invisible by matching the color of the QR code to the RFID tag antenna that makes the tag antenna hard to detect and eventually can be used in security applications. Examining QR Code Vs Barcode, it is noted that barcodes have been the standard for many years. However, when comparing QR Code Vs Barcode, it is easy to see why barcodes are being replaced by the newer QR codes. These QR Codes are becoming more common place due to their greater storage potential and ease of being read by a scanner. Damaged data can be restored from a QR Code but not from a barcode. Thus QR Codes are more reliable. The works that are strictly associated to this thesis are, Damri Samretwit et al [4], have invented an image or picture which is superimposed to that of two dimensional QR code. This QR eliminate error while reading, but further it contains raucous data,which reduces the reading capability. In this paper, taking into account the constraints, the features dimension for dissimilar locations of the overlaid image are performed. Md.Wahedul Islam et al [5], have proposed a unique DWT-SVD basedstenographic technique. A QR-code monitors the instability of the singular-values of DWT chunks. It has message rescue of maximum 85 percent. Gonzalo J.Garateguy et al [2], have established the concept of QR images, and normal method to embed QR codes into images. Zhou Wang et al [8], have proposed the methods for measuring perceptual image quality by attempted to quantify the reflectivity of errors between a distorted image and a reference image. Zachi Baharav et al [1], have implemented a method which merges the QR codes to that of color images but has some distortion. Peter Kieseberg et al, have initiates to analyze QR Codes and they attack based on human and automated systems. This paper aims at evolving a well-organized technique for embedding the color QR codes on the logos and images. The market demands small size, low cost, and environmentally compatible RFID tags. That excludes devices consuming power from batteries. Both semiconductor based tags and SAW tags can be read remotely, and both are small in size. They do not require maintenance and their life-time is limited only by the usual product time of the circuitry. However, SAW RFID tags and passive semiconductor
RFID tags are based on fundamentally different physical principles. In this section, we compare in detail these 2 approaches.

\section{A. Power Issues in SAW Tags and in IC Tags}

The main feature of SAW RFID tags is that they do not use any autonomous power supply such as batteries. Moreover, they do not include any such circuitry that would need to be powered. SAW tags are passive devices that merely reflect the request signal. This results in a linear operation at any signal level, even at a very low one. The signal energy of the SAW tag response must of course be sufficiently high for the reader to be able to receive it, which is determined by the signal-to- noise level. However, using multiple readings and matched-to-signal detection, tag signals with power below the noise level can be read. The total power radiated by the reader is typically on the order of $10 \mathrm{~mW}$. For highspeed long-read-range applications, only a fraction of a microwatt is needed at the tag position [3]. This is the typical power level to which human beings will be exposed when in proximity of SAW tag systems. It is about a hundred times lower than the radiation exposure generated by mobile phones. RFID systems based on semiconductor chips use an IC to receive and detect the signal sent by the reader, as well as to subsequently decode the signal and generate the response. The functional blocks of a typical IC tag include power accumulation, computation, and communication. The main feature of IC semiconductor tags is that they must include a proper DC power source for correct operation. The so-called passive IC RFID tags that do not carry a battery are obliged to take this power from the RF request signal. The main part of the signal sent by the reader is used to power the IC and only a small modulation of this signal is used for transmission of data. Rectifier circuitry is used to extract sufficient power from the radio signal. The rectifier converts the signal into DC for storage in a capacitor and, ultimately, for powering the chip. The reading of the tag is performed using a predetermined protocol and is only possible if the necessary DC power level is maintained throughout the entire request cycle. Therefore, a minimum critical power of about $100 \mu \mathrm{W}$ must be received continuously by the tag antenna during the entire decoding period of the tag's signal [2]. Below this signal threshold, rectification is not 
possible. This power restriction is imposed by the physics of semiconductors and thus is fundamental. For SAW tags, on the other hand, no threshold exists because they are linear passive devices. They generate a response at all power levels, usually orders of magnitude lower than what is required for IC tags.

\section{QR CODE}

\section{A. Bascis of QR Code}

QR code is expansion of bar code. It is 2-D matrix rule slightly of 1-D bar code. It saving details by division light and dark composition in the matrix. QR codes stretch engagement is ten percent of customary barcode. So it is store the fact in many ways. There are four data modes. They are: binary, numeric, alphanumeric and Kanji. The fundamental form of QR code is set in the figure 2

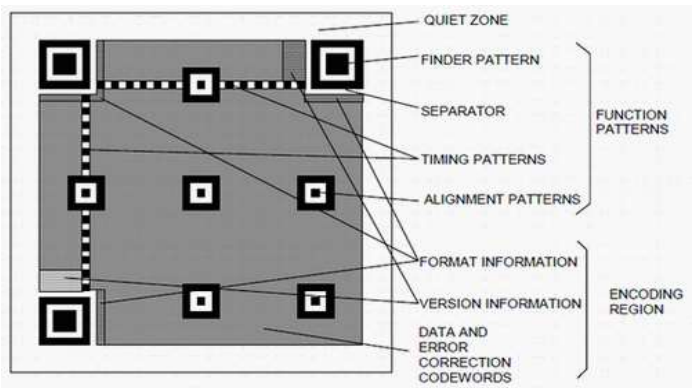

Fig 2: basic structure of QR Code

$\mathrm{QR}$ codes are made by the $\mathrm{QR}$ code generator and are decode by $\mathrm{QR}$ code scanner. The report revival from $\mathrm{QR}$ code is build the picture of the QR code. The three stairs are performed:

Step 1: Examine raw bits from the image and perform error alteration

Step2: Decode the facts and sets unrefined data into structured objects

Step3: Execute action on relevant data i.e. opening the URL

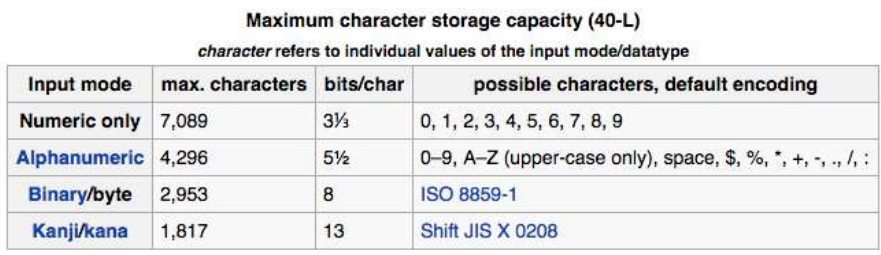

Fig 3:data modes

\section{B. Genetic Algorithm}

It is a search-based optimisation approach supported on the code of Genetics and Natural Selection. It is repeatedly utilized to detect optimal or approximateoptimal output. Genetic algorithm is division of major division of estimation known as Evolutionary Computation. The advantages of GA are: The varied advantages which have produced them extremely well-known. These contains:

- neither want any derived facts (cannot accessible for more true issues).

- Is Quicker and more effective as associated to the common ways.

- Has very well equal abilities.

- Optimized continuous and discrete functions and also various issues.

- Give a record of "good" solutions and not just a single solution

\section{PROPOSED ARCHITECTURE}

The planned system offers the idea to fix QR images and data. It is extremely secure architecture for QR code generation. To execute fusion channel code by merging two methods, Bit and Block based. Ultimately fixing color QR code into distinct images. The aimed schema wins few issues give in the modern system.

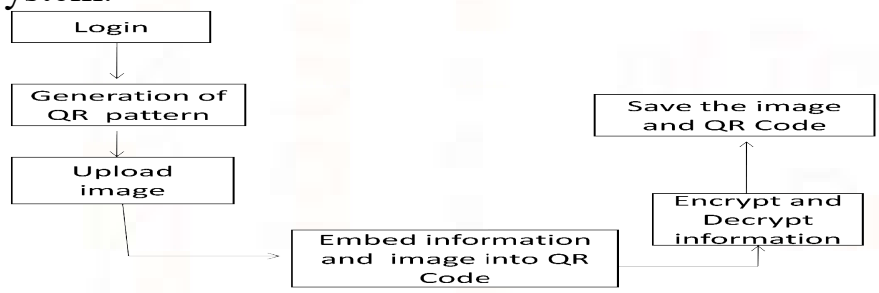

Fig 4: Proposed architecture

\section{A. Generation of QR Pattern}

In the designed procedure 4-m version contain 90 symbols with 33 number of units ,512 data pieces, 64 data passwords and 18 error alteration codeword's in each bars are applied in QR code generated. The error alteration level with $15 \%$ of data restoration

Data anatomy

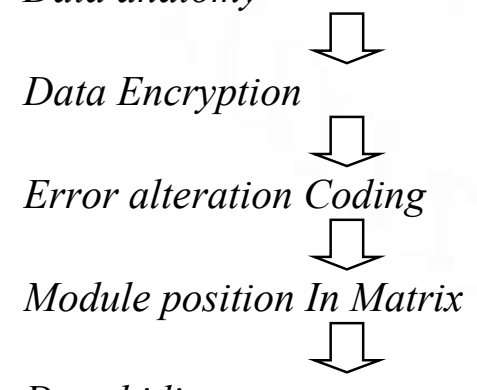

Data hiding<smiles>C1CC2CCC2C1</smiles>

format \& Version Information 


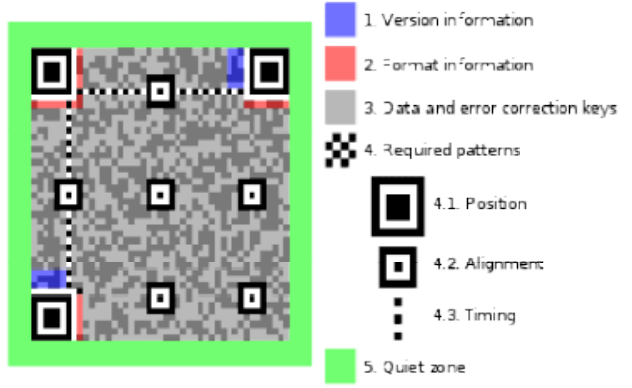

Fig 5:Generation of QR pattern

\section{B. Encoding Proceedure}

$>$ Data anatomy: A QR code enciphers orders of words and assume the maximum primary mode.

$>$ Data Encryption: This break up the order of fragments into data rule period every 8 bits extensive.

$>$ Error alteration Coding: split up the data rule period into lowered bars.

$>$ Framework conclusive: The data, error alteration code words are fabricated in the form of bars change to binary.

$>$ Module position In Matrix: put the bits in $\mathrm{QR}$ matrix. Evaluate the separators, finder, timing, patterns alignment.

$>$ Data hiding: It examines 8 hide patterns. Then audits for 4 fine principles and have a lesser quantity of excellent score.

$>$ format \& Version Information: final stage to calculate format and version information by measure pixels in definite areas.

\section{Decoding Proceedure}

The steps to decode the QR codes are as follows:

$>$ Place and get an images of the codes. Identify the Dark and Light both Modules and array of $0 \square \mathrm{s}$ and $1 \square \mathrm{s}$.

$>$ scan the structure of Version Information and discharge the hiding by XOR operation, the ciphering bit position with the Mask Pattern.

$>$ AS per the placement governs of model and recover the information and error alteration codeword's and scan the characters.

$>$ Find errors using the error alteration codeword's equal to the Level data. If any error, corrected using certain conception.

$>$ finally, decipher the Data Characters in concord with the Mode and outcome is captured.

\section{Generation of Color QR Code}

The seven stairs are repetitive and whole bit is changed into square shapes. Each bit is assigned with the pixel areas. Atlast the colors maroon is set to all the bits and pixel areas. By fusing the maroon colors and $\mathrm{QR}$ code is obtained the color. The blending of Pixel areas maroon and black in color chart.

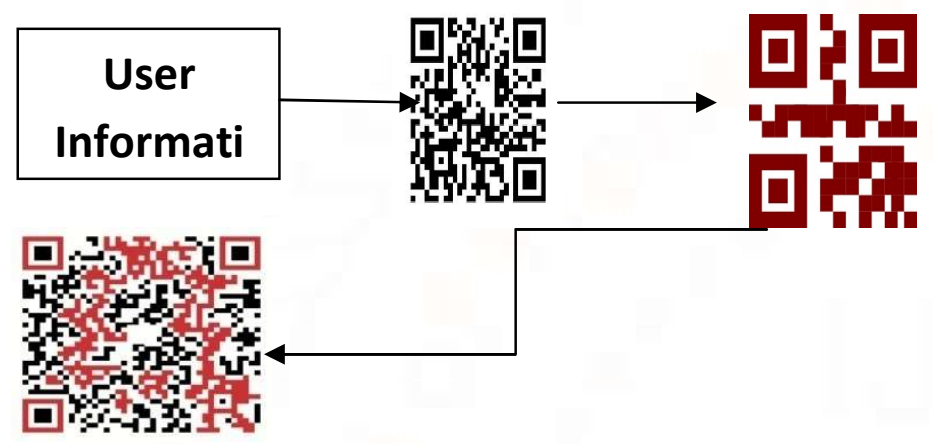

Fig 7: generation of color QR code

\section{E. Discrete Wavelet Transformation}

A wavelet is a limited dissimilarity of information image in two extensive. The basic idea wavelets are too examined. Associated with Fourier transform the wavelet has peculiar functions are infinite. The disadvantage of Fourier Transform has included the universal picture, no local Data, bad presentation and compact support. It split the image into the similarity information and split high and low resolution bandwidths. The idea is full lossless restoration and no loss in primary data.

\section{Properties of Transforms:}

Some properties of these transforms are as follows:

Decorrelation: The benefit is ejection of repeated between neighboring pixels.

$>$ Energy compression: capability of a transformation design.

$>$ Orthogonally: primary advantage of these transforms in orthogonal.

\section{EMBEDDING QR CODE IN COLOR IMAGE}

The Least Significant Bit (LSB Technique) is a insertion way and simple technique for embedding data in a graphical image file. The insertion way is all pixel changed by an all message bit. The $50 \%$ of risk 
is the message may similar with $\mathrm{LSB}^{\mathrm{ce}} \mathrm{s}$ of the hide image. Thus $50 \%$ of LSB ${ }^{e e}$ is possible to alter ensues only in the bit which is least significant, thus care the other more significant bits unchanged. It not affects the original image visibility. it is popular method. ever, it is highly vulnerable to strikes. some image handling such as cropping, force changes for any improvement such as histogram equalization, contrast stretching, addition of noise etc will kill the embedded message. The method apart from LSB technique are complex, even though they are robust to most strikes. We can save the private information such as Credit card details, ATM PIN, income tax data, salary statement, passport information etc in an invisible type. These type of data is to be protected in a method that only legal user only able to be recover it. LSB is a greater solution. Least Significant Bit encipher is the simplest techniques utilized for hidden private or personal information in digital images. The gray scale bitmap (BMP) of LSB is every byte ( 8 bits) in an image, a hidden data capacity $1 / 8$ th of the secrete image can be stored. finally, substituting of each bit in hidden data to all the LSB. For a 24-bit color image as the hide image, since 3 bytes for each pixel, 3 bits of data stored in every pixel, so that range can be highly by 3 times to store and created it 3/8 of the hide image range. If the information is embedded in text and personal message of range $1 / 7$ th grayscale screen image can be saved in 24-bit color image as screen a text message range $3 / 7$ can be embedded. The secret information is embedded in screen image can be an image (grayscale, binary or color image) text, audio etc. the range of secrete information variety of embedding a screen used.

\section{CONCLUSION}

In this Paper, a well-organized technique is used for embedding the color $\mathrm{QR}$ codes on the images. The results of this project obtain $95 \%$ and above recognition rate and better accuracy. It also shows that this method is the fastest one, having relatively great data capacity that is the color QR code with three times the data capacity than that of black and white QR codes. The use of DWT increases the correctness of reading the $\mathrm{QR}$ codes faster. Usually, all corporation uses both $\mathrm{QR}$ code and image for the safety purposes. In future we can store information, video, audio etc in QR code enhanced resolution and data capability.

\section{References}

1. Baharav Z and Kakarala R., ,Visually Significant QR Codes: Image Blending and Statistical Analysis $\square$, in Proceedings of the IEEE International Conference on Multimedia and Expo, pp. 1-6, 2013.

2. Garateguy G J,Arce G R, Lau D L, and Villarreal O P.,QR Images: Optimized Image Embedding in QR Codes国, IEEE Transactions on Image Processing, Vol.23, No. 7, pp.2842-2853,2014.

3. NANDHINI.S," PERFORMANCE EVALUATION OF EMBEDDED COLOR QR CODES ON LOGOS" 2017 Third International Conference On Science Technology Engineering and Management (ICONSTEM)

4. Wahedul Islam Md, and SaifalZahir., „A Novel QR Code GuidedImage StenographicTechnique?, in Proceedings of the IEEE International Conference on Consumer Electronics, pp.586-587, 2013.

5. Wang Z, Bovik A C, Sheikh H R, and Simoncelli E P. „Image Quality Assessment: From Error Visibility to Structural Similarity?, IEEE Transactions on Image Processing, Vol. 13, No. 4,pp. 600-612, 2004

6. D. Betancourt, M. Barahona, K. Haase, G. Schmith, A. Hübler and F. Ellinger Senior Member ${ }^{66}$ Design of printed chipless-RFID tags with QR-code appearance based on Genetic Algorithm "IEEE TRANSACTIONS ON ANTENNAS AND PROPAGATION, VOL. XX, NO. X, XXX 2017

7. Victor P. Plessky, Senior Member, IEEE, and Leonhard M. Reindl, Member, IEEE' Review on SAW RFID Tags" IEEE Transactions on Ultrasonics, Ferroelectrics, and Frequency Control, vol. 57, no. 3, March 2010 